
Not for reproduction, distribution or commercial use.

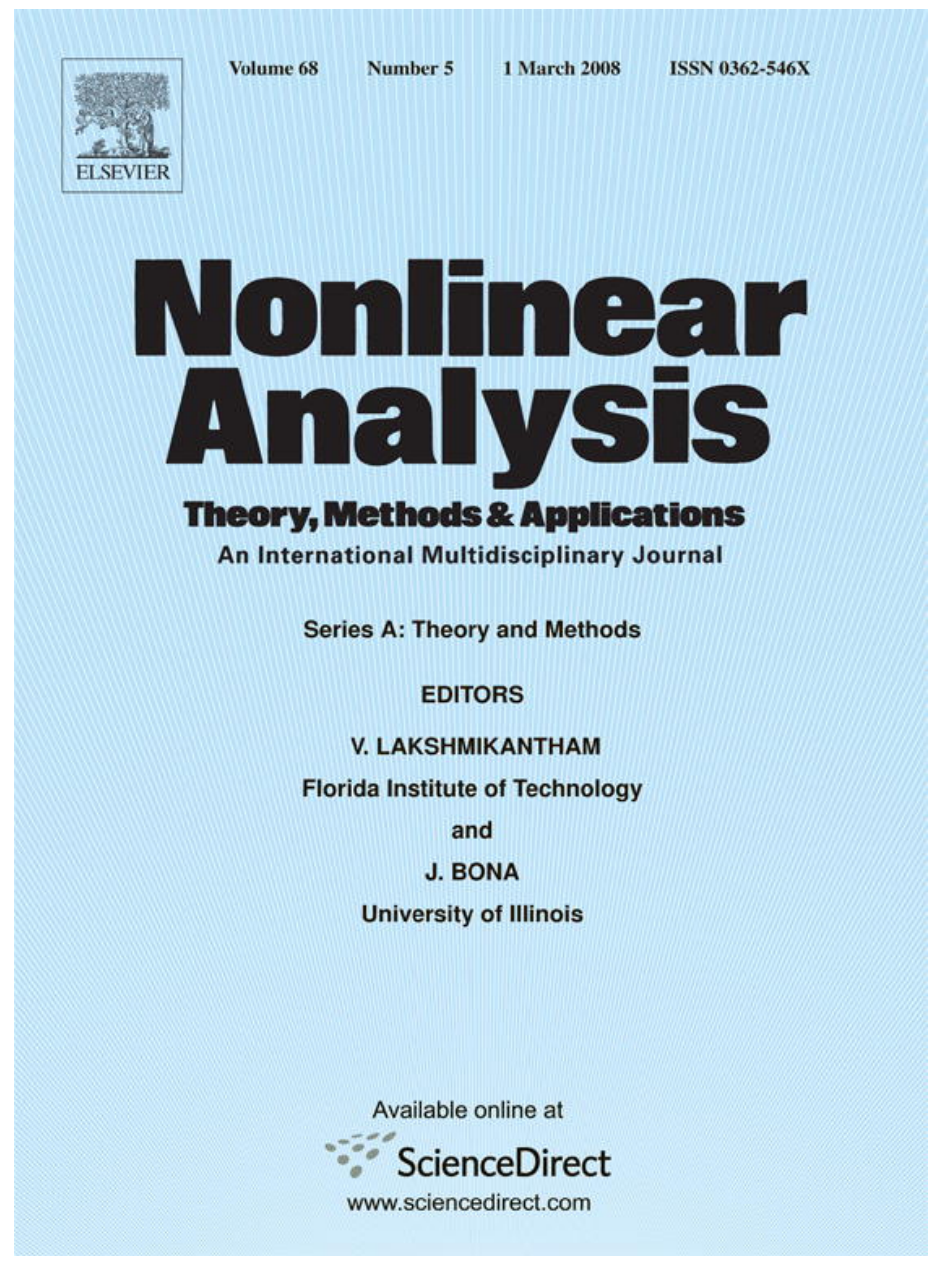

This article was published in an Elsevier journal. The attached copy

is furnished to the author for non-commercial research and education use, including for instruction at the author's institution, sharing with colleagues and providing to institution administration.

Other uses, including reproduction and distribution, or selling or licensing copies, or posting to personal, institutional or third party websites are prohibited.

In most cases authors are permitted to post their version of the article (e.g. in Word or Tex form) to their personal website or institutional repository. Authors requiring further information regarding Elsevier's archiving and manuscript policies are encouraged to visit: 


\title{
Robust exponential stabilization for large-scale uncertain impulsive systems with coupling time-delays ${ }^{\sqrt{k}}$
}

\author{
Bin Liu ${ }^{\mathrm{a}, *}$, Kok Lay Teo ${ }^{\mathrm{b}}$, Xinzhi Liu ${ }^{\mathrm{c}}$ \\ ${ }^{a}$ College of Science, Chongqing Jiaotong University, Chongqing 400074, China \\ ${ }^{\mathrm{b}}$ Department of Mathematics and Statistics, Curtin University of Technology, Perth, Western Australia, Australia \\ ${ }^{\mathrm{c}}$ Department of Applied Mathematics, University of Waterloo, Waterloo, Ontario N2L 3G1, Canada
}

Received 13 June 2006; accepted 6 December 2006

\begin{abstract}
In this paper, we aim to study robust exponential stabilization for a large-scale uncertain impulsive system with coupling time-delays. Furthermore, we also provide an estimation of the rate of convergence of exponential stabilization. By utilizing the Lyapunov method and Razumikhin technique, we shall design the feedback hybrid controllers in terms of linear matrix inequalities under which the robust exponential stability is achieved for a closed-loop large-scale uncertain impulsive system with coupling time-delays. Moreover, we shall also use the results obtained to design impulsive controllers for a large-scale uncertain continuous system under which the closed-loop continuous system achieves robust and exponential stability. To illustrate our results, one example is solved.
\end{abstract}

(C) 2007 Elsevier Ltd. All rights reserved.

Keywords: Large-scale uncertain impulsive system; Robust exponential stabilization; Decay rate; Lyapunov function; Linear matrix inequality (LMI); Coupling time-delay; Hybrid controller; Impulsive controller

\section{Introduction}

It is recognized that the theory of impulsive systems provides a natural framework for the mathematical modelling of many real world phenomena. Significant progress has been achieved in the theory of impulsive systems in recent years. For example, see [1-5,24-29] and references therein.

From the literature, we note that the stability property of a practical engineering system may be affected by various factors. The main factors are: (i) uncertainties and (ii) time-delays. In order to deal with these undesirable factors, the robust stability theory has become a promising research topic for impulsive systems. Uncertainties often occur due to modelling mismatches, measurement errors, approximations and channel noises, etc. On the other hand, time-delays occur commonly in practical engineering systems due to the congestion of the network traffic and the fact that the switching and spreading speed of the hardware and circuit implementation is finite. Moreover, time-delays presented in many real systems are difficult to know a priori and are time varying. Recently, some

\footnotetext{
This work was supported by the Post Doctoral Foundation of China (20060390247) and NSERC-Canada.

* Corresponding author.

E-mail address: oliverliu78@163.com (B. Liu).
} 
robust stability results for impulsive hybrid systems with uncertainty or with time-delays have been established in [6-12,28-33]. Moreover, robust stability and stabilization of uncertain continuous systems have also been extensively studied, see [19-23] and references therein. However, the corresponding theory for large-scale impulsive systems with uncertainties and time-delays has not been fully developed. To our knowledge, no investigation has been carried out for large-scale nonlinear impulsive systems with uncertainties and time-delays.

In this paper we aim to study the robust exponential stability of a large-scale uncertain impulsive system with coupling time-delays. Based on these stability results, we can design feedback controllers by which the corresponding closed-loop large-scale uncertain impulsive system with time-delays is robust and exponentially stable. By utilizing the ideas developed in [9-12] and the Razumikhin-type stability results established for functional differential equations [7], we shall derive several criteria, under each of which robust exponential stabilization is achieved for a closed-loop large-scale uncertain impulsive system with coupling time-delays. These controllers are expressed in terms of Linear Matrix Inequalities (LMIs). Thus, the solutions of the LMIs give rise to impulsive controllers for a large-scale uncertain continuous system under which the closed-loop continuous system is robust and exponentially stable.

The organization of this paper is as follows. In Section 2, we state the formulation of the problem and give some preliminaries. In Section 3, robust exponential stability criteria are established. These criteria can be easily used for the design of a feedback controller. For illustration, some representative examples are given in Section 4. Section 5 concludes the paper.

\section{Preliminaries and problem formulation}

Let $R^{n}$ denote the $n$-dimensional Euclidean space. Let $R_{+}=[0,+\infty), \mathbb{N}=\{0,1,2, \ldots$,$\} and \|\cdot\|$ stands for the Euclidean norm in $R^{n}$. Let $I$ be the identity matrix. The matrix $M>(\geq,<, \leq) 0$ means that $M$ is a symmetric positive definite (positive-semidefinite, negative definite, negative-semidefinite) matrix. Denote $\lambda_{\max }(\cdot)\left(\lambda_{\min }(\cdot)\right)$ as the maximum (minimum) eigenvalue of the matrix $(\cdot)$.

Consider a large-scale uncertain impulsive system consisting of $N$ impulsive subsystems with linear coupling time-delays:

$$
\begin{aligned}
& \dot{x}_{i}(t)=A_{i} x_{i}(t)+f_{i}\left(t, x_{i}(t)\right)+\sum_{j=1}^{N} B_{i j} x_{j}\left(t-\tau_{j}(t)\right)+u_{c i}(t), \quad t \in\left(t_{k}, t_{k+1}\right], \\
& \Delta x_{i}(t)=\left(C_{i k}-I\right) x_{i}(t)+u_{d i}(t), \quad t=t_{k}, k \in \mathbb{N}, i=1,2, \ldots, N
\end{aligned}
$$

where $x_{i}=\left(x_{i 1}, x_{i 2}, \ldots, x_{i n}\right)^{\mathrm{T}} \in R^{n}$, represents the state vector of the $i$ th subsystem; $\Delta x_{i}\left(t_{k}\right)=x\left(t_{k}^{+}\right)-x\left(t_{k}\right)$; $f_{i}: R_{+} \times R^{n} \rightarrow R^{n}$ is a smooth nonlinear vector function with $f_{i}(t, 0) \equiv 0 ; A_{i}, C_{i k} \in R^{n \times n}$ are the interval matrices with $A_{i} \in N\left[A_{i 1}, A_{i 2}\right]$ and $C_{i k} \in N\left[C_{i k_{1}}, C_{i k_{2}}\right]$, where $N[X, Y]=\left\{\left(x_{i j}\right) \in R^{n \times n}: u_{i j} \leq x_{i j} \leq v_{i j}\right\}$ for $X=\left(u_{i j}\right)_{n \times n}$ and $Y=\left(v_{i j}\right)_{n \times n} ; B_{i j} \in R^{n \times n}$ are the coupling coefficient matrices with $B_{i j} \in N\left[B_{i j_{1}}, B_{i j_{2}}\right]$; $\left(u_{c i}, u_{d i}\right)$ are the control inputs with $u_{c i}(t) \in U_{c} \subseteq R^{n}, u_{d i} \in U_{d} \subseteq R^{n}$. Here, we assume that $u_{c i}(\cdot)$ and $u_{d i}(\cdot)$ are restricted to the class of admissible inputs consisting of measurable functions $\left(u_{c i}(t), u_{d i}(t)\right) \in U=\left(U_{c}, U_{d}\right)$ for all $t \geq 0$, where the constraint set $U$ is given with $(0,0) \in U ; \tau_{j}(\cdot)$ is the coupling time-delay function which represents the delay of the signal transmitted from the $j$ th subsystem to $i$ th subsystem; and the sequence of impulsive instances $\left\{t_{k}\right\}$ satisfies $0 \leq t_{0}<t_{1}<t_{2}<\cdots$, with $\lim _{k \rightarrow \infty} t_{k}=\infty$.

Remark 2.1. In (1), the controllers $u_{c i}$ and $\left\{u_{d i}\left(t_{k}\right), k \in \mathbb{N}\right\}$ are said to be the continuous controller and the impulsive controller, respectively. The combined controller $\left(u_{c i}, u_{d i}\right)$ is said to be the hybrid controller. If $C_{i k_{1}}=C_{i k_{2}}=I$ and $u_{c i}=0$, then (1) can be regarded as an uncertain continuous large-scale system being controlled by impulsive controllers $\left\{u_{d i}\left(t_{k}\right), k \in \mathbb{N}\right\}$.

By [9], one can rewrite the system (1) as:

$$
\begin{aligned}
& \dot{x}_{i}=\left(A_{i 0}+\Delta A_{i}\right) x_{i}+\frac{\partial f_{i}\left(t, d_{i}\right)}{\partial x_{i}} x_{i}+\sum_{j=1}^{N}\left(B_{i j_{0}}+\Delta B_{i j}\right) x_{j}\left(t-\tau_{j}(t)\right)+u_{c i}, \quad t \in\left(t_{k}, t_{k+1}\right], \\
& \Delta x_{i}=\left(C_{i k_{0}}-I+\Delta C_{i k}\right) x_{i}(t)+u_{d i}, \quad t=t_{k}, k \in \mathbb{N}, i=1,2, \ldots, N
\end{aligned}
$$


where $f_{i}\left(t, x_{i}\right)-f_{i}(t, 0)=\frac{\partial f_{i}\left(t, d_{i}\right)}{\partial x_{i}} x_{i}, d_{i}=\theta_{i} x_{i}, 0 \leq \theta_{i} \leq 1$, and $A_{i}=A_{i 0}+\Delta A_{i}, B_{i j}=B_{i j_{0}}+\Delta B_{i j}, C_{i k}=$ $C_{i k_{0}}+\Delta C_{i k}$.

Again by [9], it follows that for any $X \in N\left[X_{1}, X_{2}\right], X$ can be formulated as:

$$
X=X_{0}+\Delta X=X_{0}+E \Sigma F,
$$

where $X_{0}=\frac{1}{2}\left(X_{1}+X_{2}\right), H=\frac{1}{2}\left(X_{2}-X_{1}\right)=\left(h_{i j}\right)_{n \times n}$,

$$
\begin{aligned}
& E \cdot E^{\mathrm{T}}=\operatorname{diag}\left\{\sum_{j=1}^{n} h_{1 j}, \ldots, \sum_{j=1}^{n} h_{n j}\right\}, \\
& F^{\mathrm{T}} \cdot F=\operatorname{diag}\left\{\sum_{j=1}^{n} h_{j 1}, \ldots, \sum_{j=1}^{n} h_{j n}\right\}, \\
& \Sigma \in \Sigma^{*}=\left\{\Sigma \in R^{n^{2} \times n^{2}}: \Sigma=\operatorname{diag}\left(\epsilon_{11}, \ldots, \epsilon_{n n}\right),\left|\epsilon_{i j}\right| \leq 1 ; i, j=1,2, \ldots, n .\right\} .
\end{aligned}
$$

Definition 2.1. The large-scale uncertain impulsive system (1) is said to be robustly exponentially stable with decay rate $\alpha$ by feedback controllers $\left(u_{c i}, u_{d i}\right)=\left(K_{c i} x_{i}, K_{d i} x_{i}\right)$, if, for any initial condition $\phi \in C\left[\left[t_{0}-\tau, t_{0}\right], R^{n N}\right]$, and every $A_{i} \in N\left[A_{i 1}, A_{i 2}\right], B_{i j} \in N\left[B_{i j_{1}}, B_{i j_{2}}\right], C_{i k} \in N\left[C_{i k_{1}}, C_{i k_{2}}\right]$, and time-delays $\tau_{j}(t)$ with $-\tau \leq \tau_{j}(t) \leq 0$, the trivial solution $x=0$ of the closed-loop system (1) is exponentially stable, in the sense that there exist two positive numbers $\alpha>0, K>0$, such that

$$
\|x(t)\| \leq K\|\phi\| \mathrm{e}^{-\alpha t}, \quad t \geq 0,
$$

where $x(t)=\left(x_{1}^{\mathrm{T}}(t), x_{2}^{\mathrm{T}}(t), \ldots, x_{N}^{\mathrm{T}}(t)\right)^{\mathrm{T}}$, and for $s \in\left[t_{0}-\tau, t_{0}\right]: x(s)=\phi(s)=\left(\phi_{1}^{\mathrm{T}}(s), \ldots, \phi_{N}^{\mathrm{T}}(s)\right)^{\mathrm{T}} \in$ $R^{n N}, \phi_{i}(s) \in R^{n}$, and $\|\phi\|^{2}=\sum_{i=1}^{N}\left\|\phi_{i}\right\|^{2},\left\|\phi_{i}\right\|=\sup _{t_{0}-\tau \leq s \leq t_{0}}\left\{\left\|\phi_{i}(s)\right\|\right\}$.

The main objective of this paper is to design feedback controllers $\left(u_{c i}, u_{d i}\right)=\left(K_{c i} x_{i}, K_{d i} x_{i}\right)$ or impulsive controllers $\left\{u_{d i}\left(t_{k}\right)=K_{d i} x_{i}\left(t_{k}\right), k \in \mathbb{N}\right\}$ under which the corresponding closed-loop system (1) is robust and exponentially stable.

Assumption 1. There exist nonnegative constants $r_{i} \geq 0, i, j=1,2, \ldots, N$, such that for all $t \in R_{+}, 0 \leq \theta_{i} \leq 1$ and $x_{i} \in R^{n}$,

$$
\left\|\frac{\partial f_{i}\left(t, d_{i}\right)}{\partial x_{i}}\right\| \leq r_{i}, \quad i=1,2, \ldots, N .
$$

Assumption 2. Assume that

$\left[\begin{array}{lll}\Delta A_{i} & \Delta B_{i j} & \Delta C_{i k}\end{array}\right]=E \Sigma\left[\begin{array}{lll}F_{A_{i}} & F_{i j} & F_{i_{k}}\end{array}\right], \quad i, j=1,2, \ldots, N, k \in \mathbb{N}$,

where $E, F_{A_{i}}, F_{i j}, F_{i_{k}}$ are known matrices and $\Sigma \in \Sigma^{*}$.

Lemma 2.1 ([13]). Let $E \in R^{n \times n^{2}}, F \in R^{n^{2} \times n}$ and $Y \in R^{n \times n}$ a symmetric matrix. Then, for any $\Sigma \in \Sigma^{*}$,

$$
Y+E \Sigma F+F^{\mathrm{T}} \Sigma E^{\mathrm{T}}<0
$$

holds if and only if there exists a positive constant $\epsilon>0$ such that

$$
Y+\epsilon E E^{\mathrm{T}}+\epsilon^{-1} F^{\mathrm{T}} F<0 .
$$

Lemma 2.2 ([16]). Let $E \in R^{n \times n^{2}}$ and $F \in R^{n^{2} \times n}$. Then, for any $\Sigma \in \Sigma^{*}$ and any positive constant $\xi>0$,

$$
E \Sigma F+F^{\mathrm{T}} \Sigma E^{\mathrm{T}} \leq \xi E E^{\mathrm{T}}+\xi^{-1} F^{\mathrm{T}} F .
$$


Lemma 2.3 ([12] (Halanay Lemma)). Let $m \in C\left[R, R_{+}\right]$be a scalar positive function such that

$$
D^{+} m(t) \leq-a_{1} m(t)+a_{2} \bar{m}(t), \quad t \geq t_{0},
$$

where $a_{1}>a_{2}>0$ and $\bar{m}(t)=\sup _{t-\tau \leq s \leq t}\{m(s)\}$. Then, there exists a constant $\alpha>0$ such that for all $t \geq t_{0}$,

$$
m(t) \leq \bar{m}\left(t_{0}\right) \mathrm{e}^{-\alpha\left(t-t_{0}\right)},
$$

where $\alpha>0$ satisfying $\alpha-a_{1}+a_{2} \mathrm{e}^{\alpha \tau}=0$.

\section{Robust exponential stabilization}

In this section, we establish some robust exponential stability criteria for the large-scale uncertain impulsive systems with coupling time-delays. These robust exponential stability criteria will be shown to be expressed as LMIs. Then, we can design the robust exponentially stable feedback controllers through solving these LMIs.

Theorem 3.1. Let $\left(u_{c i}, u_{d i}\right)=\left(K_{c i} x_{i}, K_{d i} x_{i}\right)$ and Assumptions 1 and 2 be satisfied. Assume that there exist positive definite matrices $P_{i} \in R^{n \times n}$ and constants $\gamma_{k}>0, \epsilon_{i j}>0, \varepsilon_{i}>0, \alpha_{i}<0, \beta_{i k}>0, i, j=1,2, \ldots, N, k \in \mathbb{N}$, such that the following conditions are satisfied:

(i) there exist some positive constants $v_{i}>0, \mu_{i}>0, i=1,2, \ldots, N$, such that

$$
v_{i} I \leq P_{i} \leq \mu_{i} I, \quad i=1,2, \ldots, N
$$

(ii) for $i=1,2, \ldots, N$, the following matrix inequalities hold:

$$
\left(\begin{array}{cccccc}
\Psi_{i}\left(\tilde{A}_{i 0}\right)-\alpha_{i} P_{i} & P_{i} B_{i 1_{0}} & \cdots & P_{i} B_{i N_{0}} & P_{i} E & F_{A_{i}}^{\mathrm{T}} \\
B_{i 1_{0}}^{\mathrm{T}} P_{i} & -\epsilon_{i 1} I & \cdots & 0 & 0 & F_{i 1}^{\mathrm{T}} \\
\vdots & \vdots & \ddots & \vdots & \vdots & \vdots \\
B_{i N_{0}}^{\mathrm{T}} P_{i} & 0 & \cdots & -\epsilon_{i N} I & 0 & F_{i N}^{\mathrm{T}} \\
E^{\mathrm{T}} P_{i} & 0 & \cdots & 0 & -\varepsilon_{i}^{-1} I & 0 \\
F_{A_{i}} & F_{i 1} & \cdots & F_{i N} & 0 & -\varepsilon_{i} I
\end{array}\right)<0
$$

where $\tilde{A}_{i 0}=A_{i 0}+K_{c i}, \Psi_{i}\left(\tilde{A}_{i 0}\right)=P_{i} \tilde{A}_{i 0}+\tilde{A}_{i 0}^{\mathrm{T}} P_{i}+2 r_{i} \sqrt{\frac{\mu_{i}}{v_{i}}} P_{i}$, and $\alpha_{i}, \epsilon_{i j}$ satisfy

$$
-\max _{1 \leq i \leq N}\left\{\alpha_{i}\right\}>\max _{1 \leq i \leq N}\left\{\frac{1}{v_{i}} \sum_{j=1}^{N} \epsilon_{j i}\right\} ;
$$

(iii) for $i=1,2, \ldots, N, k \in \mathbb{N}$, the following matrix inequalities hold:

$$
\left(\begin{array}{ccc}
\tilde{C}_{i k_{0}}^{\mathrm{T}} P_{i} \tilde{C}_{i k_{0}}-\beta_{i k} P_{i} & \tilde{C}_{i k_{0}}^{\mathrm{T}} P_{i} E & F_{i_{k}}^{\mathrm{T}} \\
E^{\mathrm{T}} P_{i} \tilde{C}_{i k_{0}} & -\gamma_{k}^{-1} I & 0 \\
F_{i_{k}} & 0 & -\left(\mu_{i}\left\|E^{\mathrm{T}} E\right\|+\gamma_{k}^{-1}\right) I
\end{array}\right)<0,
$$

where $\tilde{C}_{i k_{0}}=C_{i k_{0}}+K_{d i}$;

(iv) there exists a positive constant $\delta>1$ such that

$$
\tau \delta \leq \inf _{k \in \mathbb{N}}\left\{t_{k+1}-t_{k}\right\}
$$

and

$$
M \triangleq \sup _{k \in \mathbb{N}}\left\{\beta_{k}, \mathrm{e}^{\sigma \tau}\right\}<\mathrm{e}^{\delta \tau \sigma},
$$

where $\beta_{k}=\max _{1 \leq i \leq N}\left\{\beta_{k i}\right\}$, and $\sigma$ satisfies equation

$$
\sigma+a+b \mathrm{e}^{\sigma \tau}=0
$$

where $a=\max _{1 \leq i \leq N}\left\{\alpha_{i}\right\}<0$, and $b=\max _{1 \leq i \leq N}\left\{\frac{1}{v_{i}} \sum_{j=1}^{N} \epsilon_{j i}\right\}$. 
Then, the closed-loop large-scale uncertain impulsive system (2) is robustly exponentially stable with decay rate $\frac{1}{2}\left\{\sigma-\frac{\ln M}{\delta \tau}\right\}$.

Proof. Let the Lyapunov function be defined by

$$
V(t)=\sum_{i=1}^{N} x_{i}^{\mathrm{T}}(t) P_{i} x_{i}(t)
$$

Let $\tilde{A}_{i}=A_{i}+K_{c i}$. For $t \in\left[t_{k}, t_{k+1}\right)$, taking the time Dini derivative of $V(t)$ along (2) gives

$$
D^{+} V(t)=\sum_{i=1}^{N}\left\{2 x_{i}^{\mathrm{T}}(t) P_{i}\left(\tilde{A}_{i} x_{i}(t)+\frac{\partial f_{i}\left(t, d_{i}\right)}{\partial x_{i}} x_{i}(t)+\sum_{j=1}^{N} B_{i j} x_{j}\left(t-\tau_{j}(t)\right)\right)\right\}
$$

By condition (i) and Assumption 1, we get

$$
\begin{aligned}
2 x_{i}^{\mathrm{T}}(t) P_{i} \frac{\partial f_{i}\left(t, d_{i}\right)}{\partial x_{i}} x_{i}(t) & \leq 2\left\|x_{i}^{\mathrm{T}}(t) P_{i}^{\frac{1}{2}}\right\| \cdot\left\|P_{i}^{\frac{1}{2}} \frac{\partial f_{i}\left(t, d_{i}\right)}{\partial x_{i}} x_{i}(t)\right\| \\
& =2 \sqrt{x_{i}^{\mathrm{T}}(t) P_{i} x_{i}(t)} \sqrt{x_{i}^{\mathrm{T}}(t) \frac{\partial f_{i}\left(t, d_{i}\right)}{\partial x_{i}}} P_{i} \frac{\partial f_{i}\left(t, d_{i}\right)}{\partial x_{i}} x_{i}(t) \\
& \leq 2 r_{i} \sqrt{\frac{\lambda \max \left(P_{i}\right)}{\lambda_{\min }\left(P_{i}\right)}} x_{i}^{\mathrm{T}}(t) P_{i} x_{i}(t) \leq 2 r_{i} \sqrt{\frac{\mu_{i}}{v_{i}}} x_{i}^{\mathrm{T}}(t) P_{i} x_{i}(t) .
\end{aligned}
$$

It follows from (20) and (21) that, for $t \in\left[t_{k}, t_{k+1}\right)$,

$$
\begin{aligned}
D^{+} V(t) \leq & \sum_{i=1}^{N}\left\{x_{i}^{\mathrm{T}}\left[P_{i} \tilde{A}_{i}+\tilde{A}_{i}^{\mathrm{T}} P_{i}+2 r_{i} \sqrt{\frac{\mu_{i}}{v_{i}}} P_{i}\right] x_{i}+\sum_{j=1}^{N} 2 x_{i}^{\mathrm{T}}(t) P_{i} B_{i j} x_{j}\left(t-\tau_{j}(t)\right)\right\} \\
= & \sum_{i=1}^{N}\left(\begin{array}{c}
x_{i}(t) \\
x_{1}\left(t-\tau_{1}(t)\right) \\
\vdots \\
x_{N}\left(t-\tau_{N}(t)\right)
\end{array}\right)\left(\begin{array}{cccc}
\Psi_{i}\left(\tilde{A}_{i}\right) & P_{i} B_{i 1} & \cdots & P_{i} B_{i N} \\
B_{i 1}^{\mathrm{T}} P_{i} & -\epsilon_{i 1} I & \cdots & 0 \\
\vdots & \vdots & \ddots & \vdots \\
B_{i N}^{\mathrm{T}} P_{i} & 0 & \cdots & -\epsilon_{i N} I
\end{array}\right)\left(\begin{array}{c}
x_{i}(t) \\
x_{1}\left(t-\tau_{1}(t)\right) \\
\vdots \\
x_{N}\left(t-\tau_{N}(t)\right)
\end{array}\right) \\
& +\sum_{i=1}^{N} \sum_{j=1}^{N} \epsilon_{i j} x_{j}^{\mathrm{T}}\left(t-\tau_{j}(t)\right) x_{j}\left(t-\tau_{j}(t)\right)
\end{aligned}
$$

where $\Psi_{i}\left(\tilde{A}_{i}\right)=P_{i} \tilde{A}_{i}+\tilde{A}_{i}^{\mathrm{T}} P_{i}+2 r_{i} \sqrt{\frac{\mu_{i}}{v_{i}}} P_{i}$.

Let $\tilde{A}_{i 0}=A_{i 0}+K_{c i}$. By (6), then

$$
\begin{aligned}
& \left(\begin{array}{cccc}
\Psi_{i}\left(\tilde{A}_{i}\right) & P_{i} B_{i 1} & \ldots & P_{i} B_{i N} \\
B_{i 1}^{\mathrm{T}} P_{i} & -\epsilon_{i 1} I & \ldots & 0 \\
\vdots & \vdots & \ddots & \vdots \\
B_{i N}^{\mathrm{T}} P_{i} & 0 & \cdots & -\epsilon_{i N} I
\end{array}\right)=\left(\begin{array}{cccc}
\Psi_{i}\left(\tilde{A}_{i 0}\right) & P_{i} B_{i 1_{0}} & \cdots & P_{i} B_{i N_{0}} \\
B_{i 1_{0}}^{\mathrm{T}} P_{i} & -\epsilon_{i 1} I & \cdots & 0 \\
\vdots & \vdots & \ddots & \vdots \\
B_{i N_{0}}^{\mathrm{T}} P_{i} & 0 & \cdots & -\epsilon_{i N} I
\end{array}\right) \\
& +\left(\begin{array}{cccc}
P_{i} E \Sigma F_{A_{i}}+F_{A_{i}}^{\mathrm{T}} \Sigma^{\mathrm{T}} E^{\mathrm{T}} P_{i} & P_{i} E \Sigma F_{i 1} & \cdots & P_{i} E \Sigma F_{i N} \\
F_{i 1}^{\mathrm{T}} \Sigma^{\mathrm{T}} E^{\mathrm{T}} P_{i} & 0 & \cdots & 0 \\
\vdots & \vdots & \ddots & \vdots \\
F_{i N}^{\mathrm{T}} \Sigma^{\mathrm{T}} E^{\mathrm{T}} P_{i} & 0 & \cdots & 0
\end{array}\right)
\end{aligned}
$$




$$
=\left(\begin{array}{cccc}
\Psi_{i}\left(\tilde{A}_{i 0}\right) & P_{i} B_{i 1_{0}} & \cdots & P_{i} B_{i N_{0}} \\
B_{i 1_{0}}^{\mathrm{T}} P_{i} & -\epsilon_{i 1} I & \cdots & 0 \\
\vdots & \vdots & \ddots & \vdots \\
B_{i N_{0}}^{\mathrm{T}} P_{i} & 0 & \cdots & -\epsilon_{i N} I
\end{array}\right)+\left(\begin{array}{c}
P_{i} E \\
0 \\
\vdots \\
0
\end{array}\right) \Sigma\left(\begin{array}{c}
F_{A_{i}}^{\mathrm{T}} \\
F_{i 1}^{\mathrm{T}} \\
\vdots \\
F_{i N}^{\mathrm{T}}
\end{array}\right)^{\mathrm{T}}+\left(\begin{array}{c}
F_{A_{i}}^{\mathrm{T}} \\
F_{i 1}^{\mathrm{T}} \\
\vdots \\
F_{i N}^{\mathrm{T}}
\end{array}\right) \Sigma^{\mathrm{T}}\left(\begin{array}{c}
P_{i} E \\
0 \\
\vdots \\
0
\end{array}\right)^{\mathrm{T}}
$$

By Lemma 2.1, it follows that

$$
\left(\begin{array}{cccc}
\Psi_{i}\left(\tilde{A}_{i}\right)-\alpha_{i} P_{i} & P_{i} B_{i 1} & \cdots & P_{i} B_{i N} \\
B_{i 1}^{\mathrm{T}} P_{i} & -\epsilon_{i 1} I & \cdots & 0 \\
\vdots & \vdots & \ddots & \vdots \\
B_{i N}^{\mathrm{T}} P_{i} & 0 & \cdots & -\epsilon_{i N} I
\end{array}\right)<0
$$

holds if and only if there exists a positive constant $\varepsilon_{i}>0$ such that

$$
\left(\begin{array}{cccc}
\Psi_{i}\left(\tilde{A}_{i 0}\right)-\alpha_{i} P_{i} & P_{i} B_{i 1_{0}} & \cdots & P_{i} B_{i N_{0}} \\
B_{i 1_{0}}^{\mathrm{T}} P_{i} & -\epsilon_{i 1} I & \cdots & 0 \\
\vdots & \vdots & \ddots & \vdots \\
B_{i N_{0}}^{\mathrm{T}} P_{i} & 0 & \cdots & -\epsilon_{i N} I
\end{array}\right)+\varepsilon_{i}\left(\begin{array}{c}
P_{i} E \\
0 \\
\vdots \\
0
\end{array}\right)\left(\begin{array}{c}
P_{i} E \\
0 \\
\vdots \\
0
\end{array}\right)^{\mathrm{T}}+\varepsilon_{i}^{-1}\left(\begin{array}{c}
F_{A_{i}}^{\mathrm{T}} \\
F_{i 1}^{\mathrm{T}} \\
\vdots \\
F_{i N}^{\mathrm{T}}
\end{array}\right)\left(\begin{array}{c}
F_{A_{i}}^{\mathrm{T}} \\
F_{i 1}^{\mathrm{T}} \\
\vdots \\
F_{i N}^{\mathrm{T}}
\end{array}\right)^{\mathrm{T}}<0 .
$$

By the Schur Complementary Theorem [14], it follows that, for $i=1,2, \ldots, N,(25)$ is equivalent to

$$
\left(\begin{array}{cccccc}
\Psi_{i}\left(\tilde{A}_{i 0}\right)-\alpha_{i} P_{i} & P_{i} B_{i 1_{0}} & \cdots & P_{i} B_{i N_{0}} & P_{i} E & F_{A_{i}}^{\mathrm{T}} \\
B_{i 1_{0}}^{\mathrm{T}} P_{i} & -\epsilon_{i 1} I & \cdots & 0 & 0 & F_{i 1}^{\mathrm{T}} \\
\vdots & \vdots & \ddots & \vdots & \vdots & \vdots \\
B_{i N_{0}}^{\mathrm{T}} P_{i} & 0 & \cdots & -\epsilon_{i N} I & 0 & F_{i N}^{\mathrm{T}} \\
E^{\mathrm{T}} P_{i} & 0 & \cdots & 0 & -\varepsilon_{i}^{-1} I & 0 \\
F_{A_{i}} & F_{i 1} & \cdots & F_{i N} & 0 & -\varepsilon_{i} I
\end{array}\right)<0 .
$$

For $t \in\left[t_{k}, t_{k+1}\right.$ ), we see that, by condition (ii), (26) implies that

$$
\begin{aligned}
D^{+} V(t) & \leq \sum_{i=1}^{N} \alpha_{i} x_{i}^{\mathrm{T}} P_{i} x_{i}+\sum_{i=1}^{N} \sum_{j=1}^{N} \epsilon_{i j} x_{j}^{\mathrm{T}}\left(t-\tau_{j}(t)\right) x_{j}\left(t-\tau_{j}(t)\right) \\
& \leq \max _{1 \leq i \leq N}\left\{\alpha_{i}\right\} \sum_{i=1}^{N} x_{i}^{\mathrm{T}} P_{i} x_{i}+\max _{1 \leq j \leq N}\left\{\frac{1}{v_{i}} \sum_{j=1}^{N} \epsilon_{j i}\right\} \sum_{i=1}^{N} x_{i}^{\mathrm{T}}\left(t-\tau_{i}(t)\right) P_{i} x_{i}\left(t-\tau_{i}(t)\right) \\
& \leq \max _{1 \leq i \leq N}\left\{\alpha_{i}\right\} \cdot V+\max _{1 \leq i \leq N}\left\{\frac{1}{v_{i}} \sum_{j=1}^{N} \epsilon_{j i}\right\} \cdot \bar{V}(t) .
\end{aligned}
$$

Hence, by Lemma 2.3 and condition (ii), it follows that, for $t \in\left[t_{k}, t_{k+1}\right.$ ), there exists a constant $\sigma>0$ such that

$$
V(t) \leq \bar{V}\left(t_{k}\right) \mathrm{e}^{-\sigma\left(t-t_{k}\right)},
$$

where $\bar{V}\left(t_{k}\right)=\sup _{t_{k}-\tau \leq s \leq t_{k}}\{V(s)\}$, and $\sigma>0$ satisfies (18).

Let $\tilde{C}_{i k}=C_{i k}+K_{d i}$ and $\tilde{C}_{i k_{0}}=C_{i k_{0}}+K_{d i}$. When $t=t_{k}$, we can use Lemma 2.2 to show that, for some positive constants $\gamma_{k}>0, k \in \mathbb{N}$,

$$
\begin{aligned}
V\left(t_{k}\right) & =\sum_{i=1}^{N} x_{i}^{\mathrm{T}}\left(t_{k}\right) P_{i} x_{i}\left(t_{k}\right)=\sum_{i=1}^{N} x_{i}^{\mathrm{T}}\left(t_{k}^{-}\right)\left[\left(\tilde{C}_{i k_{0}}+E \Sigma F_{i_{k}}\right)^{\mathrm{T}} P_{i}\left(\tilde{C}_{i k_{0}}+E \Sigma F_{i_{k}}\right)\right] x_{i}\left(t_{k}^{-}\right) \\
& =\sum_{i=1}^{N} x_{i}^{\mathrm{T}}\left(t_{k}^{-}\right)\left[\tilde{C}_{i k_{0}}^{\mathrm{T}} P_{i} \tilde{C}_{i k_{0}}+F_{i_{k}}^{\mathrm{T}} \Sigma^{\mathrm{T}} E^{\mathrm{T}} P_{i} \tilde{C}_{i k_{0}}+\tilde{C}_{i k_{0}}^{\mathrm{T}} P_{i} E \Sigma F_{i_{k}}+F_{i_{k}}^{\mathrm{T}} \Sigma^{\mathrm{T}} E^{\mathrm{T}} P_{i} E \Sigma F_{i_{k}}\right] x_{i}\left(t_{k}^{-}\right)
\end{aligned}
$$




$$
\begin{aligned}
& \leq \sum_{i=1}^{N} x_{i}^{\mathrm{T}}\left(t_{k}^{-}\right)\left\{\tilde{C}_{i k_{0}}^{\mathrm{T}} P_{i} \tilde{C}_{i k_{0}}+\gamma_{k} \tilde{C}_{i k_{0}}^{\mathrm{T}} P_{i} E E^{\mathrm{T}} P_{i} \tilde{C}_{i k_{0}}+\gamma_{k}^{-1} F_{i_{k}}^{\mathrm{T}} F_{i_{k}}+\left(\mu_{i}\left\|E^{\mathrm{T}} E\right\|\right) F_{i_{k}}^{\mathrm{T}} F_{i_{k}}\right\} x_{i}\left(t_{k}^{-}\right) \\
& =\sum_{i=1}^{N} x_{i}^{\mathrm{T}}\left(t_{k}^{-}\right)\left\{\tilde{C}_{i k_{0}}^{\mathrm{T}} P_{i} \tilde{C}_{i k_{0}}+\gamma_{k} \tilde{C}_{i k_{0}}^{\mathrm{T}} P_{i} E E^{\mathrm{T}} P_{i} \tilde{C}_{i k_{0}}+\left(\mu_{i}\left\|E^{\mathrm{T}} E\right\|+\gamma_{k}^{-1}\right) F_{i_{k}}^{\mathrm{T}} F_{i_{k}}\right\} x_{i}\left(t_{k}^{-}\right) .
\end{aligned}
$$

By the Schur Complementary Theorem, it holds that, for $i=1,2, \ldots, N$,

$$
\tilde{C}_{i k_{0}}^{\mathrm{T}} P_{i} \tilde{C}_{i k_{0}}+\gamma_{k} \tilde{C}_{i k_{0}}^{\mathrm{T}} P_{i} E E^{\mathrm{T}} P_{i} \tilde{C}_{i k_{0}}+\left(\mu_{i}\left\|E^{\mathrm{T}} E\right\|+\gamma_{k}^{-1}\right) F_{i_{k}}^{\mathrm{T}} F_{i_{k}}-\beta_{k i} P_{i}<0
$$

is equivalent to the following matrix inequalities:

$$
\left(\begin{array}{ccc}
\tilde{C}_{i k_{0}}^{\mathrm{T}} P_{i} \tilde{C}_{i k_{0}}-\beta_{k i} P_{i} & \tilde{C}_{i k_{0}}^{\mathrm{T}} P_{i} E & F_{i_{k}}^{\mathrm{T}} \\
E^{\mathrm{T}} P_{i} \tilde{C}_{i k_{0}} & -\gamma_{k}^{-1} I & 0 \\
F_{i_{k}} & 0 & -\left(\mu_{i}\left\|E^{\mathrm{T}} E\right\|+\gamma_{k}^{-1}\right) I
\end{array}\right)<0 .
$$

Hence, by condition (iii), we obtain

$$
V\left(t_{k}\right)<\sum_{i=1}^{N} \beta_{k i} x_{i}^{\mathrm{T}}\left(t_{k}^{-}\right) P_{i} x_{i}\left(t_{k}^{-}\right) \leq \beta_{k} \sum_{i=1}^{N} x_{i}^{\mathrm{T}}\left(t_{k}^{-}\right) P_{i} x_{i}\left(t_{k}^{-}\right)=\beta_{k} V\left(t_{k}^{-}\right),
$$

where $\beta_{k}=\max _{1 \leq i \leq N}\left\{\beta_{k i}\right\}, k \in \mathbb{N}$.

In the following, we shall show, by induction, that

$$
V(t) \leq \mu M^{k-1}\|\phi\|^{2} \mathrm{e}^{-\sigma\left(t-t_{0}\right)}, \quad t \in\left[t_{k-1}, t_{k}\right), k \in \mathbb{N},
$$

where $\mu=\max _{1 \leq i \leq N}\left\{\mu_{i}\right\}$.

When $k=1$, since for all $t \in\left[t_{0}-\tau, t_{0}\right]$,

$$
\left\|x_{i}(t)\right\|=\left\|\phi_{i}(t)\right\| \leq\left\|\phi_{i}\right\|=\sup _{t_{0}-\tau \leq t \leq t_{0}}\left\|\phi_{i}(t)\right\|
$$

we get

$$
V(t) \leq \max _{1 \leq i \leq N}\left\{\lambda_{\max }\left(P_{i}\right)\right\} \cdot \sum_{i=1}^{N}\left\|x_{i}(t)\right\|^{2} \leq \mu \cdot\|\phi\|^{2}, \quad t \in\left[t_{0}-\tau, t_{0}\right],
$$

where $\|\phi\|^{2}=\sum_{i=1}^{N}\left\|\phi_{i}\right\|^{2}$.

Hence, we have

$$
\bar{V}\left(t_{0}\right) \leq \mu \cdot\|\phi\|^{2} .
$$

By (28) and (34), we get

$$
V(t) \leq \bar{V}\left(t_{0}\right) \mathrm{e}^{-\sigma\left(t-t_{0}\right)} \leq \mu \cdot\|\phi\|^{2} \mathrm{e}^{-\sigma\left(t-t_{0}\right)}=M^{0} \mu \cdot\|\phi\|^{2} \mathrm{e}^{-\sigma\left(t-t_{0}\right)}, \quad t \in\left[t_{0}, t_{1}\right) .
$$

Thus, (33) holds for $k=1$.

Now assume (33) holds for $k \leq m, m>1$. Then, we shall show that (33) holds for $k=m+1$.

By (32) and (17) and the induction assumption, we have

$$
V\left(t_{m}\right) \leq \beta_{m} V\left(t_{m}^{-}\right) \leq \mu \beta_{m} M^{m-1}\|\phi\|^{2} \mathrm{e}^{-\sigma\left(t_{m}-t_{0}\right)} \leq \mu M^{m}\|\phi\|^{2} \mathrm{e}^{-\sigma\left(t_{m}-t_{0}\right)} .
$$

Hence, by condition (iv) and (36), it follows that, for $k=m+1, t \in\left[t_{m}, t_{m+1}\right.$ ),

$$
\begin{aligned}
V(t) & \leq \bar{V}\left(t_{m}\right) \cdot \mathrm{e}^{-\sigma\left(t-t_{m}\right)}=\max _{t_{m}-\tau \leq t \leq t_{m}}\{V(t)\} \cdot \mathrm{e}^{-\sigma\left(t-t_{m}\right)} \\
& =\max \left\{\sup _{t_{m}-\tau \leq t<t_{m}}\{V(t)\}, V\left(t_{m}\right)\right\} \mathrm{e}^{-\sigma\left(t-t_{m}\right)} \\
& \leq \max \left\{\mu M^{m-1}\|\phi\|^{2} \mathrm{e}^{-\sigma\left(t_{m}-\tau-t_{0}\right)}, \mu M^{m}\|\phi\|^{2} \mathrm{e}^{-\sigma\left(t_{m}-t_{0}\right)}\right\} \mathrm{e}^{-\sigma\left(t-t_{m}\right)} \\
& =\max \left\{M^{m-1} \mathrm{e}^{\sigma \tau}, M^{m}\right\} \mu\|\phi\|^{2} \mathrm{e}^{-\sigma\left(t_{m}-t_{0}\right)} \mathrm{e}^{-\sigma\left(t-t_{m}\right)} \\
& \leq \mu M^{m}\|\phi\|^{2} \mathrm{e}^{-\sigma\left(t-t_{0}\right)} .
\end{aligned}
$$

Therefore, by the induction principle, we see that (33) holds for all $k \in \mathbb{N}$. 
Finally, we shall show that

$$
\|x(t)\| \leq K\|\phi\| \mathrm{e}^{-\alpha\left(t-t_{0}\right)}, \quad t \geq t_{0},
$$

where, $\alpha=\frac{1}{2}\left\{\sigma-\frac{\ln M}{\delta \tau}\right\}>0, K=\sqrt{\frac{\mu}{v}}$, and $v=\min _{1 \leq i \leq N}\left\{v_{i}\right\}$.

Since $\delta \tau \leq \inf _{k \in \mathbb{N}}\left\{t_{k}-t_{k-1}\right\}$, we have $k-1 \leq \frac{t_{k-1}-t_{0}}{\delta \tau}$, which implies $M^{k-1} \leq \mathrm{e}^{\frac{\ln M}{\delta \tau}\left(t_{k-1}-t_{0}\right)}$. Thus, for $t \in\left[t_{k-1}, t_{k}\right)$, we get

$$
\|x(t)\|^{2} \leq \frac{V(t)}{v} \leq \frac{\mu}{v}\|\phi\|^{2} M^{k-1} \mathrm{e}^{-\sigma\left(t-t_{0}\right)} \leq \frac{\mu}{v}\|\phi\|^{2} \mathrm{e}^{-\left(\sigma-\frac{\ln M}{\delta \tau}\right)\left(t-t_{0}\right)} .
$$

Hence, (38) holds, which implies that system (2) is robustly exponentially stable with decay rate $\frac{1}{2}\left\{\sigma-\frac{\ln M}{\delta \tau}\right\}$. The proof is complete.

Remark 3.1. Theorem 3.1 presents the robust exponential stability criteria. However, these criteria cannot be used to design the feedback control gain matrices $K_{c i}, K_{d i}, i=1,2, \ldots, N$. The following corollary aims to address this problem.

Corollary 3.1. Let Assumptions 1 and 2 be satisfied. Assume that, for given $\mu_{i}>0$ and $v_{i}>0, i=1,2, \ldots, N$, conditions (i) and (iv) of Theorem 3.1 are satisfied, while conditions (ii)-(iii) are replaced by the following conditions $\left(\right.$ ii* $\left.^{*}\right)-\left(\right.$ iii $\left.^{*}\right)$ :

(ii*) there exist matrices $Y_{c i} \in R^{n \times n}$, such that, for $i=1,2, \ldots, N$, the following LMIs hold:

$$
\left(\begin{array}{cccccc}
\Psi_{i}\left(\tilde{A}_{i 0}\right)+Y_{c i}^{\mathrm{T}}+Y_{c i}-\alpha_{i} P_{i} & P_{i} B_{i 1_{0}} & \cdots & P_{i} B_{i N_{0}} & P_{i} E & F_{A_{i}}^{\mathrm{T}} \\
B_{i 1_{0}}^{\mathrm{T}} P_{i} & -\epsilon_{i 1} I & \cdots & 0 & 0 & F_{i 1}^{\mathrm{T}} \\
\vdots & \vdots & \ddots & \vdots & \vdots & \vdots \\
B_{i N_{0}}^{\mathrm{T}} P_{i} & 0 & \cdots & -\epsilon_{i N} I & 0 & F_{i N}^{\mathrm{T}} \\
E^{\mathrm{T}} P_{i} & 0 & \cdots & 0 & -\epsilon_{i}^{-1} I & 0 \\
F_{A_{i}} & F_{i 1} & \cdots & F_{i N} & 0 & -\epsilon_{i} I
\end{array}\right)<0,
$$

where $\Psi_{i}\left(\tilde{A}_{i 0}\right)=P_{i} \tilde{A}_{i 0}+\tilde{A}_{i 0}^{\mathrm{T}} P_{i}+2 r_{i} \sqrt{\frac{\mu_{i}}{v_{i}}} P_{i}$ and $\alpha_{i}, \epsilon_{i j}$ satisfy (14);

(iii*) there exist matrices $Y_{d i} \in R^{n \times n}$, such that, for $i=1,2, \ldots, N$, the following LMIs hold:

$$
\left(\begin{array}{cccc}
C_{i k_{0}}^{\mathrm{T}} P_{i} C_{i k_{0}}+Y_{d i}^{\mathrm{T}} C_{i k_{0}}+C_{i k_{0}} Y_{d i}-\beta_{i k} P_{i} & C_{i k_{0}}^{\mathrm{T}} P_{i} E+Y_{d i}^{\mathrm{T}} E & F_{i_{k}}^{\mathrm{T}} & Y_{d i}^{\mathrm{T}} \\
E^{\mathrm{T}} P_{i} C_{i k_{0}}+E^{\mathrm{T}} Y_{d i} & -\gamma_{k}^{-1} I & 0 & 0 \\
F_{i_{k}} & 0 & -\left(\mu_{i}\left\|E^{\mathrm{T}} E\right\|+\gamma_{k}^{-1}\right) I & 0 \\
Y_{d i} & 0 & 0 & -P_{i}
\end{array}\right)<0 .
$$

Then, the large-scale uncertain impulsive system (2) with the controllers $\left(u_{c i}, u_{d i}\right)=\left(P_{i}^{-1} Y_{c i} x_{i}, P_{i}^{-1} Y_{d i} x_{i}\right)$, $i=1,2, \ldots, N$, is robustly exponentially stable with decay rate $\frac{1}{2}\left\{\sigma-\frac{\ln M}{\delta \tau}\right\}$.

Proof. Let $K_{c i}=P_{i}^{-1} Y_{c i}, K_{d i}=P_{i}^{-1} Y_{d i}, i=1,2, \ldots, N$. Then, by Theorem 3.1 and the Schur Complementary Theorem, the conditions of the theorem follows readily.

Remark 3.2. It is interesting to discuss two special cases: (i) $u_{c i}=0$ and (ii) $u_{d i}=0$. That is to say, only the continuous controllers $u_{c i}$ or only the impulsive controllers $u_{d i}$ are used to robustly and exponentially stabilize system (2). In the case of $u_{d i}=0$, i.e., $K_{d i}=0$, Theorem 3.1 and Corollary 3.1 can be used to design the continuous feedback controllers $u_{c i}=K_{c i} x_{i}, i=1,2, \ldots, N$, to robustly and exponentially stabilize the system. But when $u_{c i}=0$, i.e., $Y_{c i}=0$ or $K_{c i}=0$, it is noted that the condition for $\alpha_{i}<0$ in Theorem 3.1 and Corollary 3.1 is not satisfied. In fact, when $u_{c i}=0$, i.e., $K_{c i}=0$, and matrix $A_{i}$ is not a stable matrix, then $\alpha_{i}>0$. In the following, we will use the Razumikhin technique (see [15]) and Theorem 3.1 in [7] to solve this problem. 
Theorem 3.2. Let $u_{c i}=0, u_{d i}=K_{d i} x_{i}$ and Assumptions 1 and 2 be satisfied. Assume that there exist positive definite matrices $P_{i} \in R^{n \times n}$, and constants $\gamma_{k}>0, \epsilon_{i j}>0, \varepsilon_{i}>0, \hat{\alpha}_{i}>0,0<\beta_{i k}<1, i, j=1,2, \ldots, N, k \in \mathbb{N}$, such that

(i) there exist some positive constants $v_{i}>0, \mu_{i}>0, i=1,2, \ldots, N$, such that

$$
v_{i} I \leq P_{i} \leq \mu_{i} I, \quad i=1,2, \ldots, N ;
$$

(ii) for $k \in \mathbb{N}, i=1,2, \ldots, N$, the following matrix inequalities hold:

$$
\left(\begin{array}{cccccc}
\Psi_{i}\left(A_{i 0}\right)-\hat{\alpha}_{i} P_{i} & P_{i} B_{i 1_{0}} & \cdots & P_{i} B_{i N_{0}} & P_{i} E & F_{A_{i}}^{\mathrm{T}} \\
B_{i 1_{0}}^{\mathrm{T}} P_{i} & -\epsilon_{i 1} I & \cdots & 0 & 0 & F_{i 1}^{\mathrm{T}} \\
\vdots & \vdots & \ddots & \vdots & \vdots & \vdots \\
B_{i N_{0}}^{\mathrm{T}} P_{i} & 0 & \cdots & -\epsilon_{i N} I & 0 & F_{i N}^{\mathrm{T}} \\
E^{\mathrm{T}} P_{i} & 0 & \cdots & 0 & -\varepsilon_{i}^{-1} I & 0 \\
F_{A_{i}} & F_{i 1} & \cdots & F_{i N} & 0 & -\varepsilon_{i} I
\end{array}\right)<0,
$$

where $\Psi_{i}\left(A_{i 0}\right)=P_{i} A_{i 0}+A_{i 0}^{\mathrm{T}} P_{i}+2 r_{i} \sqrt{\frac{\mu_{i}}{v_{i}}} P_{i}$;

(iii) for $i=1,2, \ldots, N, k \in \mathbb{N}$, the following matrix inequalities hold:

$$
\left(\begin{array}{ccc}
\tilde{C}_{i k_{0}}^{\mathrm{T}} P_{i} \tilde{C}_{i k_{0}}-\beta_{i k} P_{i} & \tilde{C}_{i k_{0}}^{\mathrm{T}} P_{i} E & F_{i_{k}}^{\mathrm{T}} \\
E^{\mathrm{T}} P_{i} \tilde{C}_{i k_{0}} & -\gamma_{k}^{-1} I & 0 \\
F_{i_{k}} & 0 & -\left(\mu_{i}\left\|E^{\mathrm{T}} E\right\|+\gamma_{k}^{-1}\right) I
\end{array}\right)<0,
$$

where $\tilde{C}_{i k_{0}}=C_{i k_{0}}+K_{d i}$;

(iv) there exists a positive constant $\sigma$ with $\sigma>p$ such that

$$
\beta_{k} \leq \mathrm{e}^{-\sigma\left(t_{k+1}-t_{k}\right)},
$$

where $\beta_{k}=\max _{1 \leq i \leq N}\left\{\beta_{k i}\right\}, p=p_{1}+p_{2}, p_{1}=\max _{1 \leq i \leq N}\left\{\hat{\alpha}_{i}\right\}$,

and $p_{2}=\max _{1 \leq i \leq N}\left\{\frac{1}{v_{i}} \sum_{j=1}^{N} \epsilon_{j i}\right\}$.

Then, the closed-loop large-scale uncertain impulsive system (2) is robustly exponentially stable with decay rate $\frac{1}{2}(\sigma-p)$.

Proof. Let the Lyapunov function be defined by

$$
V(t)=\sum_{i=1}^{N} x_{i}^{\mathrm{T}}(t) P_{i} x_{i}(t)
$$

Then, by an argument similar to that used in the proof of Theorem 3.1, it follows from condition (i) that, for $t \in\left[t_{k}, t_{k+1}\right)$,

$$
\begin{aligned}
D^{+} V(t) & \leq \sum_{i=1}^{N} \hat{\alpha}_{i} x_{i}^{\mathrm{T}} P_{i} x_{i}+\sum_{i=1}^{N} \sum_{j=1}^{N} \epsilon_{i j} x_{j}^{\mathrm{T}}\left(t-\tau_{j}(t)\right) x_{j}\left(t-\tau_{j}(t)\right) \\
& \leq \max _{1 \leq i \leq N}\left\{\hat{\alpha}_{i}\right\} \sum_{i=1}^{N} x_{i}^{\mathrm{T}} P_{i} x_{i}+\max _{1 \leq j \leq N}\left\{\frac{1}{v_{i}} \sum_{j=1}^{N} \epsilon_{j i}\right\} \sum_{i=1}^{N} x_{i}^{\mathrm{T}}\left(t-\tau_{i}(t)\right) P_{i} x_{i}\left(t-\tau_{i}(t)\right) \\
& \triangleq p_{1} V(t)+p_{2} V(t-\tau(t)),
\end{aligned}
$$

where $p_{1}=\max _{1 \leq i \leq N}\left\{\hat{\alpha}_{i}\right\}, p_{2}=\max _{1 \leq i \leq N}\left\{\frac{1}{v_{i}} \sum_{j=1}^{N} \epsilon_{j i}\right\}$.

Thus, by Theorem 3.1 in [7], if $V(t+s) \leq V(t)$ for any $-\tau \leq s \leq 0$, then

$$
D^{+} V(t) \leq\left(p_{1}+p_{2}\right) V(t) \triangleq p V(t)
$$


which implies that

$$
V(t) \leq V\left(t_{k}\right) \mathrm{e}^{p\left(t-t_{k}\right)}, \quad t \in\left[t_{k}, t_{k+1}\right), k \in \mathbb{N},
$$

where $p=p_{1}+p_{2}>0$.

Let $\tilde{C}_{i k_{0}}=C_{i k_{0}}+K_{d i}$. By condition (iii), it follows from an argument similar to that used in the proof of Theorem 3.1, that

$$
V\left(t_{k}\right)<\sum_{i=1}^{N} \beta_{k i} x_{i}^{\mathrm{T}}\left(t_{k}^{-}\right) P_{i} x_{i}\left(t_{k}^{-}\right) \leq \beta_{k} \sum_{i=1}^{N} x_{i}^{\mathrm{T}}\left(t_{k}^{-}\right) P_{i} x_{i}\left(t_{k}^{-}\right)=\beta_{k} V\left(t_{k}^{-}\right),
$$

where $\beta_{k}=\max _{1 \leq i \leq N}\left\{\beta_{k i}\right\}, k \in \mathbb{N}$.

From (48) and (49), we obtain

$$
V(t) \leq \beta_{k} V\left(t_{k}^{-}\right) \mathrm{e}^{p\left(t-t_{k}\right)}=V\left(t_{k}^{-}\right) \mathrm{e}^{p\left(t-t_{k}\right)+\ln \beta_{k}}, \quad t \in\left[t_{k}, t_{k+1}\right), k \in \mathbb{N},
$$

which implies that

$$
V\left(t_{k+1}^{-}\right) \leq V\left(t_{k}^{-}\right) \mathrm{e}^{p\left(t_{k+1}-t_{k}\right)+\ln \beta_{k}} \leq V\left(t_{k}^{-}\right) \mathrm{e}^{-(\sigma-p)\left(t_{k+1}-t_{k}\right)} \leq \cdots \leq V\left(t_{0}\right) \mathrm{e}^{-(\sigma-p)\left(t_{k+1}-t_{0}\right)} .
$$

Thus, for any $t \in\left[t_{k}, t_{k+1}\right), k \in \mathbb{N}$, we obtain

$$
V(t) \leq V\left(t_{0}\right) \mathrm{e}^{-(\sigma-p)\left(t-t_{0}\right)},
$$

which implies that, for any initial condition $x(s)=\phi(s), s \in[-\tau, 0]$,

$$
\|x(t)\| \leq \sqrt{\frac{\mu}{v}}\|\phi\| \mathrm{e}^{-\alpha\left(t-t_{0}\right)} .
$$

Hence, by (53) and Theorem 3.1 in [7], system (2) with the impulsive controllers $u_{d i}=K_{d i} x_{i}, i=1,2, \ldots, N$, is robustly exponentially stable with decay rate $\frac{1}{2}(\sigma-p)$. The proof is complete.

Corollary 3.2. Let $u_{c i}=0$ and Assumptions 1 and 2 be satisfied. Assume that, for given $\mu_{i}>0$ and $v_{i}>0$, $i=1,2, \ldots, N$, conditions (i)-(ii) and (iv) of Theorem 3.2 are satisfied, while condition (iii) is replaced by the following (iii*):

(iii*) for $i=1,2, \ldots, N, k \in \mathbb{N}$, the following LMIs hold:

$$
\left(\begin{array}{cccc}
C_{i k_{0}}^{\mathrm{T}} P_{i} C_{i k_{0}}+Y_{d i}^{\mathrm{T}} C_{i k_{0}}+C_{i k_{0}} Y_{d i}-\beta_{i k} P_{i} & C_{i k_{0}}^{\mathrm{T}} P_{i} E+Y_{d i}^{\mathrm{T}} E & F_{i_{k}}^{\mathrm{T}} & Y_{d i}^{\mathrm{T}} \\
E^{\mathrm{T}} P_{i} C_{i k_{0}}+E^{\mathrm{T}} Y_{d i} & -\gamma_{k}^{-1} I & 0 & 0 \\
F_{i_{k}} & 0 & -\left(\mu_{i}\left\|E^{\mathrm{T}} E\right\|+\gamma_{k}^{-1}\right) I & 0 \\
Y_{d i} & 0 & 0 & -P_{i}
\end{array}\right)<0 .
$$

Then, the large-scale uncertain system (2) with the impulsive controllers $u_{d i}=P_{i}^{-1} Y_{d i} x_{i}, i=1,2, \ldots, N$, is robustly exponentially stable with decay rate $\frac{1}{2}(\sigma-p)$.

Proof. The conclusion follows as a direct consequence of Theorem 3.2 with $K_{d i}=P_{i}^{-1} Y_{d i}, i=1,2, \ldots, N$.

Remark 3.3. In system (1), if $C_{i k_{1}}=C_{i k_{2}}=I$ and $u_{c i}=0$, then (1) can be regarded as an uncertain continuous largescale system which is subjected to the impulsive controllers $\left\{u_{d i}\left(t_{k}\right), k \in \mathbb{N}\right\}$. In this case, $C_{i k}=I$ and Corollary 3.2 can be used to design the feedback impulsive controllers $\left\{K_{d i} x_{i}\left(t_{k}\right), k \in \mathbb{N}\right\}$ under which the closed-loop continuous uncertain system is robustly exponentially stable with decay rate $\frac{1}{2}(\sigma-p)$.

\section{Examples and simulations}

In this section, one representative example is given for illustration. Here, the numerical simulation procedure is coded and executed in the MATLAB environment. 
Example 4.1. Let the chaotic Colpitts' oscillator [17] be used as a subsystem in the large-scale uncertain system with coupling time-delays. A single Colpitts' oscillator is in the form of

$$
\left\{\begin{array}{l}
\dot{y}_{1}=\alpha y_{2} \\
\dot{y}_{2}=-\sigma\left(y_{1}+\gamma y_{2}+y_{3}\right) \\
\dot{y}_{3}=\beta\left(y_{2}+a_{1} y_{1}+a_{3} y_{1}^{3}\right)
\end{array}\right.
$$

where $\alpha, \beta, \sigma, a_{1}, \gamma, a_{3} \in R$. It is known that when the parameters $\alpha=2.4, \beta=2.2, \sigma=1, \gamma=0.252, a_{1}=1$ and $a_{3}=-0.2$, system (55) is chaotic.

Let $y=\left(y_{1}, y_{2}, y_{3}\right)^{\mathrm{T}}$. Then, the matrix form of the Colpitts' oscillator is described as:

$$
\dot{y}=A_{0} y+\varphi(y)
$$

where $A_{0}=\left(\begin{array}{ccc}0 & \alpha & 0 \\ -\sigma & -\gamma \sigma & -\sigma \\ a_{1} \beta & \beta & 0\end{array}\right)$ and $\varphi(y)=\left(\begin{array}{c}0 \\ 0 \\ a_{3} \beta y_{1}^{3}\end{array}\right)$.

Suppose that the large-scale uncertain system with coupling time-delays is given by

$$
\dot{x}_{i}=A x_{i}+\varphi\left(x_{i}\right)+\sum_{j=1}^{N} B_{i j} x_{j}\left(t-\tau_{j}(t)\right), \quad i=1,2, \ldots, N,
$$

where $x_{i}=\left(x_{i 1}, x_{i 2}, x_{i 3}\right)^{\mathrm{T}}$, the matrix $A \in N\left[A_{1}, A_{2}\right]$, where

$$
A_{1}=\left(\begin{array}{ccc}
-0.5 & \alpha-0.5 & -0.5 \\
-\sigma-0.5 & -\gamma \sigma-0.5 & -\sigma-0.5 \\
a_{1} \beta-0.5 & \beta-0.5 & -0.5
\end{array}\right) \quad \text { and } \quad A_{2}=\left(\begin{array}{ccc}
0.5 & \alpha+0.5 & 0.5 \\
-\sigma+0.5 & -\gamma \sigma+0.5 & -\sigma+0.5 \\
a_{1} \beta+0.5 & \beta+0.5 & 0.5
\end{array}\right) \text {, }
$$

while the coupling term matrices are in the form of

$$
B_{i i}=\left(\begin{array}{ccc}
0.5 & 0.5 & 0 \\
0 & 0.5 & 0.2 \\
0 & 0 & -0.5
\end{array}\right), \quad B_{i, i+1}=\left(\begin{array}{ccc}
-1.0 & -0.3 & 0 \\
0 & 0.25 & -0.1 \\
0 & 0 & 1.0
\end{array}\right), \quad B_{i, i+2}=\left(\begin{array}{ccc}
0.5 & -0.2 & 0 \\
0 & -0.75 & -0.1 \\
0 & 0 & -0.5
\end{array}\right),
$$

and $B_{N-1, N+1}=B_{N-1,1}, B_{N-1, N+2}=N_{N-1,2}, B_{N, N+1}=B_{N 1}$, and $B_{N, N+2}=B_{N 2}$.

In the following, we will design impulsive controller $u_{d i}\left(t_{k}\right)=K_{d i} x_{i}\left(t_{k}\right)$ with which the system

$$
\begin{aligned}
& \dot{x}_{i}=A x_{i}+\varphi\left(x_{i}\right)+\sum_{j=1}^{N} B_{i j} x_{j}\left(t-\tau_{j}(t)\right), \quad t \in\left(t_{k}, t_{k+1}\right], \\
& \Delta x_{i}(t)=u_{i}(t)=K_{d i}\left(x_{i}(t)-y(t)\right), \quad t=t_{k}, k \in \mathbb{N}, i=1,2, \ldots, N,
\end{aligned}
$$

is robustly and exponentially stable.

From [18,19], it follows that

$$
\left\|\frac{\partial \varphi\left(d_{i}\right)}{\partial x_{i}}\right\| \leq 5.28, \quad i=1,2, \ldots, N .
$$

To apply Corollary 3.2, we choose $\epsilon_{i j}=1, \epsilon_{i}=1, v_{i}=1, \mu_{i}=2, Y_{c i}=0$. Then, by using the LMI Tool Box within the MATLAB environment to solve the LMIs (54), for $i=1,2, \ldots, 10$, we obtain $\hat{\alpha}_{i}=6, \beta_{i k}=$ $0.01, \max _{k \in \mathbb{N}}\left\{t_{k+1}-t_{k}\right\}=0.2878$, and

$$
P_{i}=\left(\begin{array}{lll}
1.5437 & 0.3646 & 0.1371 \\
0.3646 & 1.4298 & 0.1290 \\
0.1371 & 0.1290 & 1.1520
\end{array}\right), \quad Y_{d i}=\left(\begin{array}{lll}
-1.5328 & -0.3546 & -0.1330 \\
-0.3546 & -1.4215 & -0.1264 \\
-0.1330 & -0.1264 & -1.1502
\end{array}\right) .
$$

Now, we may design the required impulsive controller $u_{d i}\left(t_{k}\right)=K_{d i} x_{i}\left(t_{k}\right)$ as follows: 


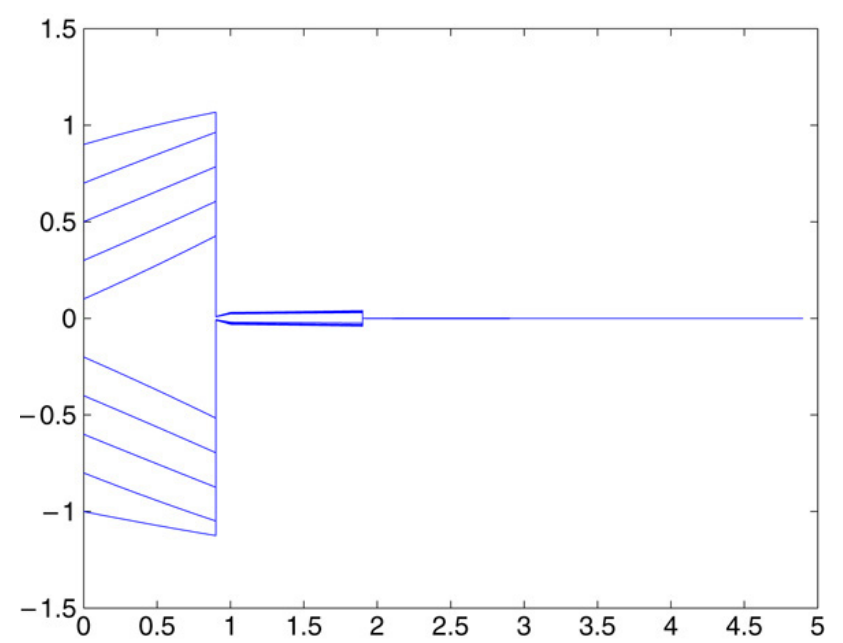

Fig. 1. The first elements of states, $x_{k 1}(t), k=1,2, \ldots, 10$.

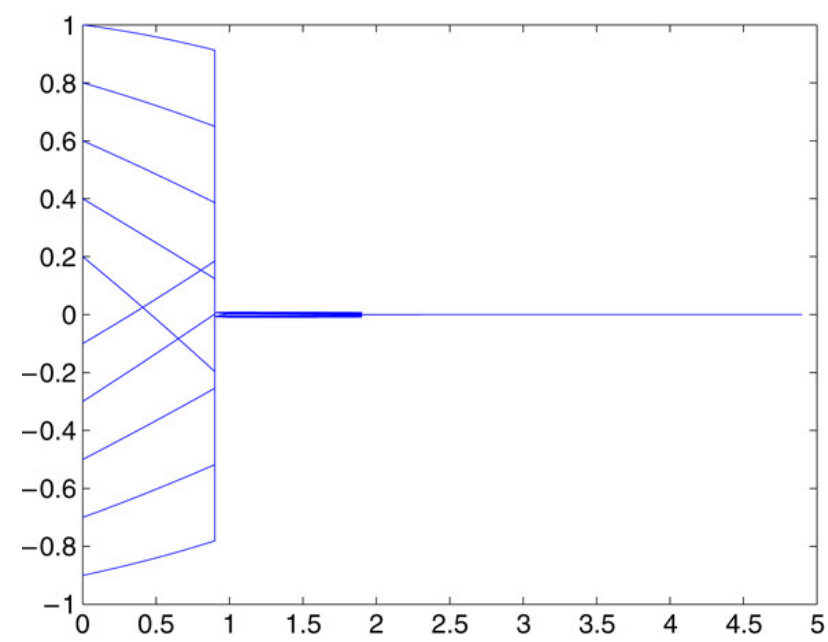

Fig. 2. The second elements of states, $x_{k 2}(t), k=1,2, \ldots, 10$.

$t_{0}=0, t_{k}-t_{k-1}=0.2, k \in \mathbb{N}$, and the impulsive control gain matrices $K_{d i}$ are:

$$
K_{d i}=P_{i}^{-1} Y_{d i}=\left(\begin{array}{ccc}
-0.9944 & 0.0053 & 0.0022 \\
0.0022 & -0.9957 & 0.0011 \\
0.0022 & 0.0011 & -0.9988
\end{array}\right), \quad i=1,2, \ldots, 10 .
$$

Thus, $p_{1}=6, p_{2}=10$. Therefore, by setting $\sigma=23$, we obtain

$$
\sigma>p=p_{1}+p_{2}, \quad \text { and } \quad \beta_{k} \leq \mathrm{e}^{-\sigma\left(t_{k+1}-t_{k}\right)} \quad k \in \mathbb{N} \text {. }
$$

Hence, by Corollary 3.2, the impulsive controller $u_{d i}\left(t_{k}\right)=K_{d i} x_{i}\left(t_{k}\right)$ designed above can achieve robust exponential stabilization for this large-scale uncertain system and its decay rate is $\frac{1}{2}\{\sigma-p\}=3.5$.

In the simulations, we choose $t_{k+1}-t_{k}=0.2, k \in \mathbb{N}$, and set, without loss of generality, the initial conditions $x_{i}(t)=c_{i}$, whenever $t \leq 0$, where $c_{i}, i=1,2, \ldots, N$, are constants. For any $A \in N\left[A_{1}, A_{2}\right]$, we set $A=A_{1}+R_{3 \times 3}$, where the matrix $R_{3 \times 3}=\left(r_{i j}\right)_{3 \times 3}$ is a $3 \times 3$ random matrix in which $0 \leq r_{i j} \leq 1$. In Figs. 1-3 we let $\tau_{j}(t) \equiv 0.1$, and we see that the large-scale uncertain system can be robustly exponentially stabilized by the controller given by (61). In Figs. 4-6 we use different time-delays: $\tau_{j}(t)=\frac{11-j}{100}, j=1,2, \ldots, 10$, then we can see that the large-scale uncertain system can still be robustly exponentially stabilized by the same controller given by (61). 


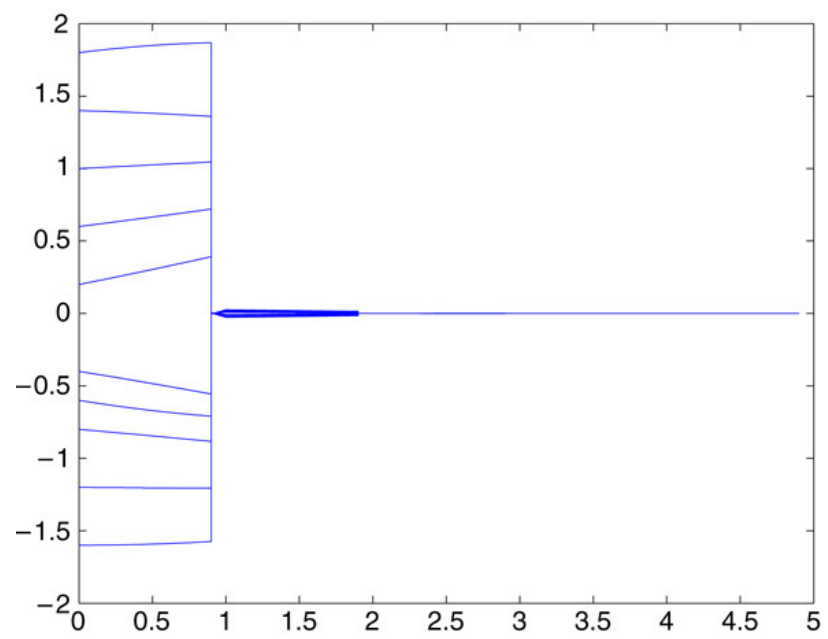

Fig. 3. The third elements of states, $x_{k 3}(t), k=1,2, \ldots, 10$.

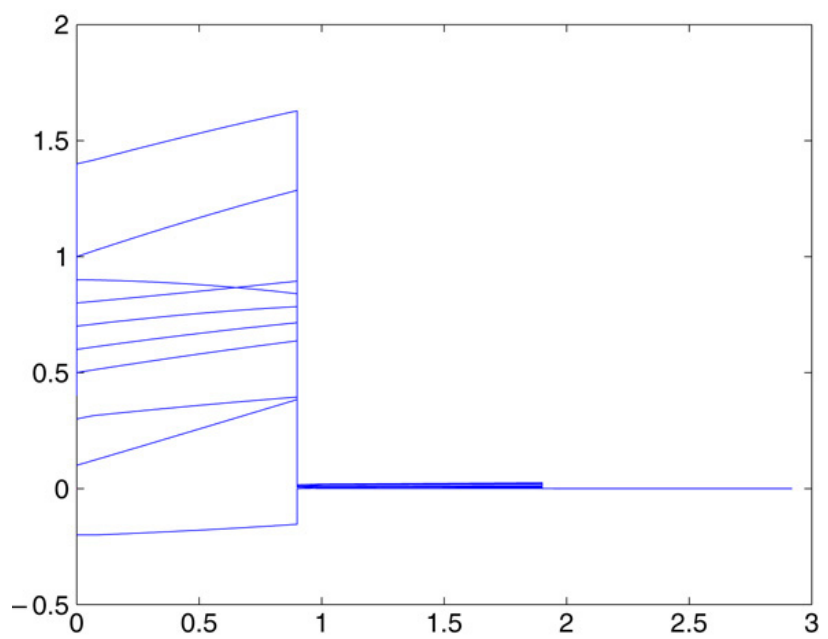

Fig. 4. The first elements of states, $x_{k 1}(t), k=1,2, \ldots, 10$.

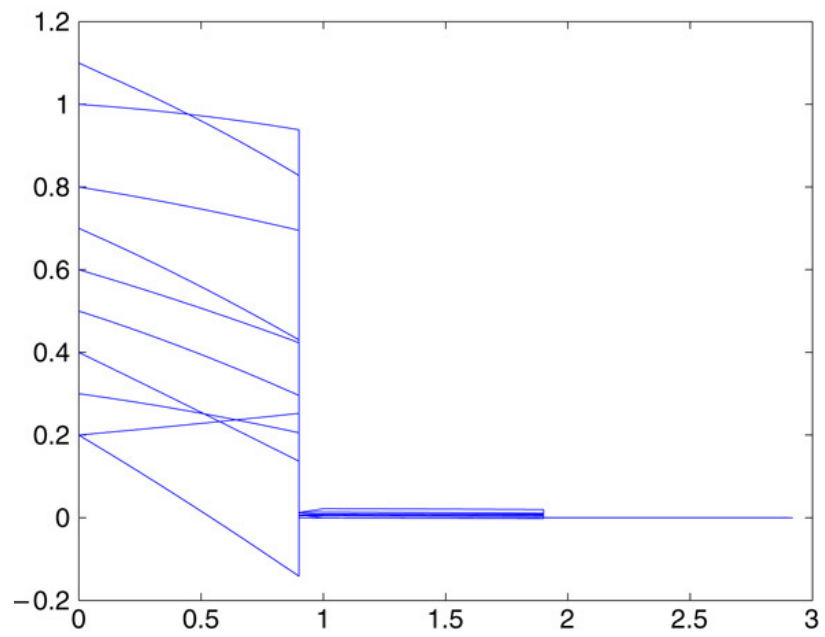

Fig. 5. The second elements of states, $x_{k 2}(t), k=1,2, \ldots, 10$. 


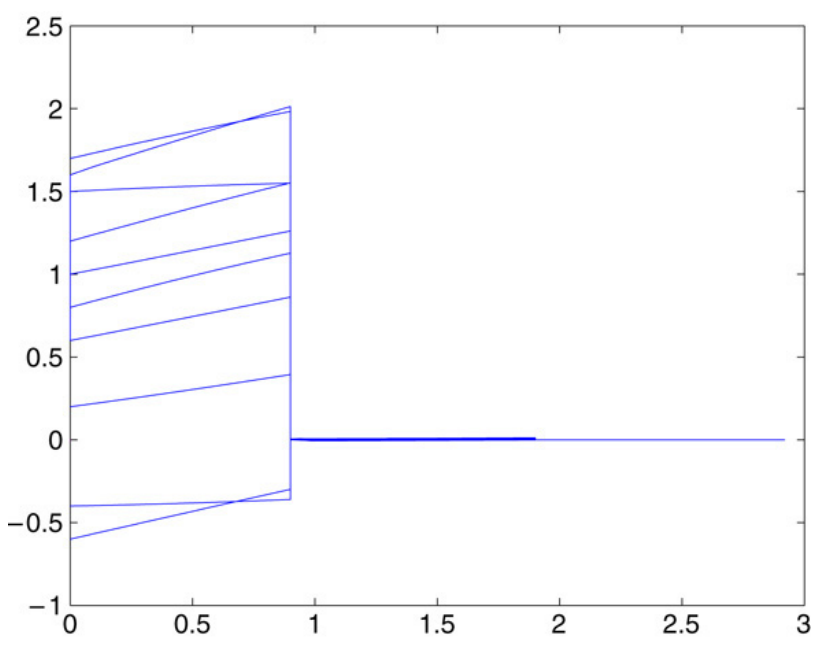

Fig. 6. The third elements of states, $x_{k 3}(t), k=1,2, \ldots, 10$.

\section{Conclusions}

The coupling of subsystems seriously affects the dynamical behaviors such as the stability of a large-scale system. Since coupling time-delay and uncertainty often occur in a practical large-scale system, it is important to investigate their effect on the stability of a large-scale system. In this paper, the robust exponential stabilization problem for a large-scale uncertain impulsive system with coupling time-delays has been studied by employing hybrid feedback control techniques and an impulsive control method. The controller so designed is robust with respect to the uncertainties in the subsystem parameters and the coupling time-delays. By using the methods of the Lyapunov function and Razumikhin technique as well as Linear Matrix Inequalities (LMIs), some simple and effective criteria for achieving robust exponential stabilization have been derived, with fast convergence rates. The decay rates are also obtained to estimate the convergence rate. These conditions are expressed in terms of LMIs. Finally, one relevant example, for impulsive controllers, has been considered and solved so as to illustrate the results obtained in the paper.

\section{References}

[1] V. Lakshmikantham, D.D. Bainov, P.S. Simeonov, Theory of Impulse Differential Equations, Singapore World Scientific, 1989.

[2] D.D. Bainov, P.S. Simeonov, Systems with Impulsive Effects: Stability Theory and Applications, Halsted Press, New York, 1989.

[3] V. Lakshmikantham, X. Liu, Stability Analysis in Terms of Two Measures, Singapore World Scientific, 1993.

[4] X. Liu, Stability results for impulsive differential systems with applications to population growth models, Dynam. Stability Syst. 9 (2) (1994) $163-174$.

[5] H. Ye, A.N. Michel, L. Hou, Stability analysis of systems with impulsive effects, IEEE Trans. Automat. Control 43 (12) (1998) $1719-1723$.

[6] G. Ballinger, X. Liu, Existence and uniqueness results for impulsive delay differential equations, DCDIS 5 (1999) 579-591.

[7] X. Liu, G. Ballinger, Uniform asymptotic stability of impulsive delay differential equations, Comput. Math. Appl. 41 (2001) 903-915.

[8] J.H. Shen, J. Yan, Razumikhin type stability theorems for impulsive functional differential equations, Nonlinear Anal. 33 (1998) $519-531$.

[9] B. Liu, X. Liu, X. Liao, Robust stability analysis of uncertain impulsive systems, J. Math. Anal. Appl. 290 (2004) 519-533.

[10] B. Liu, X. Liu, X. Liao, Stability and robust stability of quasi-linear impulsive hybrid dynamical systems, J. Math. Anal. Appl. 283 (2003) 416-430.

[11] B. Liu, X. Liu, Robust global exponential stability and estimate of decay rate of uncertain linear impulsive systems with time-delay, Rocky Moutain J. Math. 36 (2) (2006) 615-636.

[12] B. Liu, X. Liu, K.L. Teo, Razumikhin-type theorems on exponential stability of impulsive delay systems, IMA J. Appl. Math. 71 (2006) $47-61$.

[13] Y.M. Moon, P. Park, W.H. Kwon, Y.S. Lee, Delay-dependent robust stabilization of uncertain state-delayed systems, Int. J. Control 74 (14) (2001) 1447-1455.

[14] S. Boyd, L. El Ghaoui, E. Feron, V. Balakrishnan, Linear Matrix Inequality in Systems and Control Theory, SIAM, Philadelphia, 1994.

[15] J.K. Hale, S.M. Verduyn Lunel, Introduction to Functional Differential Equations, Springer, New York, 1993.

[16] I.R. Petersen, A stabilization algorithm for a class of uncertain systems, Syst. Control Lett. 8 (1987) 181-188.

[17] G. Chen, Controlling Chaos and Bifurcations in Engineering Systems, CRC Press, Boca Raton, FL, 2000.

[18] B. Liu, X. Liu, G. Chen, H. Wang, Robust impulsive synchronization of uncertain dynamical networks, IEEE Trans. Circuits Syst.-I. Regular Paper 52 (7) (2005) 1431-1441.

[19] D.T. Gavel, D.D. Siljak, Decentralized adaptive control: Structural conditions for stability, IEEE Trans. Automat. Control 34 (1989) $413-426$. 
[20] L. Bakule, J. Rodellar, Decentralized control and overlapping decomposition of mechanical systems, Int. J. Control 61 (1995) $559-587$.

[21] M.S. Mahmoud, S. Bingulac, Robust design of stabilizing controllers for interconnected time-delay systems, Automatica 34 (1998) 795-800.

[22] H.J. Gao, J. Lam, C.H. Wang, Q. Wang, Hankel norm approximation of linear systems with time-varying delay: Continuous and discrete cases, Int. J. Control 77 (17) (2004) 1520-1530.

[23] X.R. Mao, J. Lam, S.Y. Xu, H.J. Gao, Razumikhin method and exponential stability of hybrid stochastic delay interval systems, J. Math. Anal. Appl. 283 (2006) 416-430.

[24] Z.G. Li, C.B. Soh, X.H. Xu, Controllability and observability of impulsive hybrid dynamic systems, IMA J. Math. Control Inform. 16 (1999) $315-334$.

[25] Z.G. Li, Y.C. Soh, C.Y. Wen, Robust stability of a class of hybrid nonlinear systems, IEEE Trans. Automat. Control 46 (6) (2001) $897-903$.

[26] Z.G. Li, Y.C. Soh, C.Y. Wen, Switched and Impulsive Systems: Analysis, Design and Application, Springer-Verlag, ISBN: 3-540-23952-9, 2005.

[27] Z.G. Li, C.Y. Wen, Y.C. Soh, Analysis and design of impulsive control systems, IEEE Trans. Automat. Control 46 (2001) $894-899$.

[28] Y. Zhang, J.T. Sun, Stability of impulsive delay differential equations with impulses at variable times, Dynam. Syst.—An International Journal 20 (3) (2005) 323-331.

[29] Y. Zhang, J.T. Sun, Stability of impulsive linear differential equations with time delay, IEEE Trans. Circuits Syst. II-Express Briefs 52 (2005) 701-705.

[30] X. Liu, J.H. Shen, Stability theory of hybrid dynamical systems with time delays, IEEE Trans. Automat. Control 51 (4) (2006) $620-625$.

[31] P. Pepe, On the asymptotic stability of coupled delay differential and continuous time difference equations, Automatica 41 (2005) $107-112$.

[32] V.U. Phat, Robust stability and stabilizability of uncertain linear hybrid systems with state delays, IEEE Trans. Circuits Syst. II-Express Briefs 52 (2) (2005) 94-98.

[33] B. Liu, Robust stability of uncertain discrete impulsive hybrid systems, IEEE Trans. Circuits Syst. II—Express Briefs, 2007 (in press). 